\title{
Prominences on Rapidly-Rotating Solar-Type Stars
}

\author{
M. Jardine, J. Barnes, Y. Unruh and A. Collier Cameron \\ Department of Physics and Astronomy, University of St. Andrews, North \\ Haugh, St. Andrews, Fife, KY16 9SS U.K.
}

\begin{abstract}
We present $\mathrm{H} \alpha$ observations of two rapidly-rotating G2 dwarfs in the Alpha Persei cluster and of AB Dor, a young K0 dwarf. All three stars have projected rotational velocities of about $90 \mathrm{~km} \mathrm{~s}^{-1}$ and axial rotation periods ranging from 11 to 15 hours. He 520 and AB Dor show very clear transient features that move through the profile. The most likely explanation for these features is that they are huge prominences in co-rotation with the star. We also use the recently developed technique of least squares deconvolution to present simultaneous dynamic profiles of the stars' photospheric lines which show the locations of the surface spots. This technique has allowed us to extend our studies to a greater range of stars.
\end{abstract}

\section{Introduction}

Prominences on rapidly-rotating solar-like stars have been observed now for nearly 13 years (Collier Cameron and Robinson 1989a, b). They are observed as transient absorption features that move through the $\mathrm{H} \alpha$ profile. From the rate at which these features move we can find the distances of the prominences from the stellar rotation axis. Significantly, in contrast to the Sun, we find that most prominences are located at great distances (typically 2 to 6 stellar radii) from the stellar rotation axis. While similar in their temperatures and formation times to solar prominences, these prominences are larger and more massive (by two orders of magnitude) than their solar counterparts. They are also much shorter-lived, surviving for only a few days. The surface positions of the spots on these stars can also be found using the technique of Doppler Imaging (Vogt and Penrod 1983), although it is still an open question as to how stellar prominences are linked to the surface magnetic fields and star spots. On AB Dor (the most consistently-observed example) we find that spots appear in two active latitudes, one at about $30^{\circ}$ and one at about $80^{\circ}$. Recently, Donati and Collier Cameron (1997) have used the least squares deconvolution of approximately 1500 photospheric lines to produce Doppler maps both of the surface spots on $A B$ Dor and of the radial and horizontal components of the magnetic field in the bright regions. Using data obtained on the 7th and 11th of November 1995 they have also derived the surface differential rotation which is very similar to that for the Sun (in that the equator laps the pole in 120 days rather than 110 days for the Sun). This result not only places severe constraints on dynamo mechanisms for this star, but also on the possible means of destabilising the prominences. 


\section{The Observations}

Observations of the $\alpha$ Persei stars were taken using the Utrecht Echelle Spectrograph at the 4.2m William Herschel Telescope on October 25-26, 1996 and November 24-26, 1996. The magnitude of both He 699 and He 520 (i.e., $m_{v} \approx 11.5$ ) necessitated exposure times of $900 \mathrm{~s}$ for each observation. This allowed a signal to noise ratio of $40-60$ to be obtained in the raw spectra while keeping the effects of rotational blurring to a minimum. The AB Dor data were obtained using the UCL spectrograph at the Anglo Australian Telescope. This star is much brighter $\left(m_{v} \approx 7\right)$, thereby allowing a greater time resolution (i.e., shorter exposure times). In both cases, data reduction was carried out using standard Starlink packages. The resolution of both sets of H $\alpha$ dynamic spectra is $6 \mathrm{~km} \mathrm{~s}^{-1}$ per pixel and $3 \mathrm{~km} \mathrm{~s}^{-1}$ for the photospheric dynamic spectra.
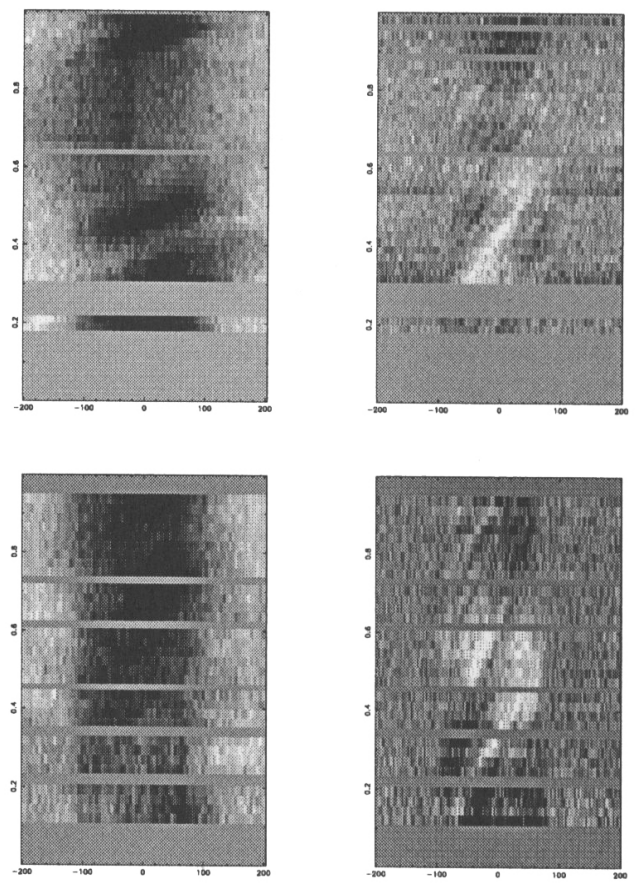

Figure 1. Phase plotted against vsini. Top and Bottom Left: He 520 and $\mathrm{He} 699 \mathrm{H} \alpha$ dynamic spectra, Top and Bottom Right: He 520 and He 699 photospheric dynamic spectra. Half-profile widths are $v \sin i=$ $86 \mathrm{~km} \mathrm{~s}^{-1}(\mathrm{He} \mathrm{520})$ and $v \sin i=94 \mathrm{~km} \mathrm{~s}^{-1}$ (He 699).

\section{Prominence Positions}

The $\mathrm{H} \alpha$ observations give us the distances of the prominences from the rotation axis which is inclined at some angle to the observer's line of sight. For a group 

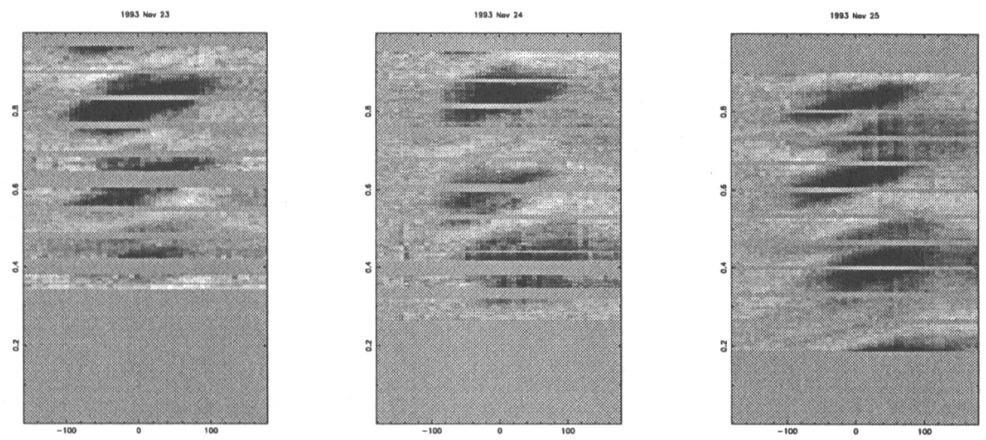

Figure 2. $\mathrm{AB}$ Dor, $\mathrm{H} \alpha$ dynamic spectra, November 23-25, 1993. Note that individual features are repeated, and show evolution from night to night ( $\left.v \sin i=91 \mathrm{~km} \mathrm{~s}^{-1}\right)$. This suggests that they are prominences, and not shorter lived features such as stellar flares.

of stars in the one cluster the inclination angle can be determined using the technique of Hendry et al. 1993 and O'Dell et al. 1994. Table 1 summarizes the prominence positions and inclinations (ranging from $60^{\circ}$ to $90^{\circ}$ ) for four $\mathrm{G}$ dwarfs in the young $(50 \mathrm{Myr})$ open cluster, $\alpha$ Persei. The age of the cluster tells us that the $\mathrm{G}$ dwarfs should lie on the zero age main sequence. Because prominences can only be observed when they cross in front of the stellar disk, for those stars with a lower inclination (where the observer is looking in at high latitudes), any prominences that are observed far from the rotation axis must be confined at high latitudes well out of the equatorial plane. This suggests that the footpoints of the magnetic field lines confining these prominences must both lie in the same hemisphere (see Ferreira 1998, these proceedings).

TABLE 1 - Alpha Persei and AB Doradus data

\begin{tabular}{ll|ccccc}
\hline Parameters & & He 520 & He 699 & He 373 & He 622 & AB Dor \\
\hline $\mathrm{P}$ & {$[\mathrm{d}]$} & 0.6079 & 0.4908 & 0.333 & 0.804 & 0.5148 \\
$v \sin i$ & {$\left[\mathrm{~km} \mathrm{~s}^{-1}\right]$} & $86 \pm 2$ & $94 \pm 4$ & 140 & 61 & 91 \\
$R_{c}$ & {$\left[R_{\odot}\right]$} & 3.0 & 2.6 & 2.0 & 3.64 & 2.5 \\
$R_{p}(\mathrm{CW} 92)$ & {$\left[R_{*}\right]$} & $3.7-5.1$ & $3.8-6.0$ & $2.0-2.8$ & 5.4 & - \\
$R_{p}(1996)$ & {$\left[R_{*}\right]$} & $2.8-3.0$ & $1.7 ; 1.3-2.4$ & - & - & - \\
$R_{p} 23$ Nov & {$\left[R_{*}\right]$} & - & - & - & - & $3.1-3.4$ \\
$R_{p} 24$ Nov & {$\left[R_{*}\right]$} & - & - & - & - & $2.6-2.9$ \\
$R_{p} 25$ Nov & {$\left[R_{*}\right]$} & - & - & - & - & $3.5-4.3$ \\
$i$ & & $90^{\circ}$ & $62^{\circ}$ & $61^{\circ}$ & $70^{\circ}$ & $60^{\circ}$ \\
\hline
\end{tabular}

For comparison, prominence positions for several Alpha Per stars were calculated from the paper by Collier Cameron and Woods (1992) (CW92). The new (1996) data allow both prominence and spot positions to be calculated for $\mathrm{He} 520$ and He 699. The dynamic $\mathrm{H} \alpha$ spectra in Figure 1 show that for He 520, dark absorption features cross the profile at a rate which shows that they lie close to the co-rotation radius, $R_{c}$. He 699 appears to show lower lying features, and 
we cannot exclude that they are at the photosphere. We, therefore, conclude that these features may indeed be active regions, rather than prominences. The dynamic photospheric spectra in Figure 1 show the underlying photospheric features (note, spots appear bright) produced by the least squares deconvolution of approximately 1000 metal lines. The deconvolution process yields a combined profile with sufficient $\mathrm{S}: \mathrm{N}$ to give significant deviations of profile distortions produced by surface inhomogeneities such as cool spots. The dynamic spectrum of He 699 shows spot features that do not cross the whole profile, indicating high latitude or polar features. He 520 seems to indicate a high latitude region of spots also. By comparison, high latitude features are also seen on AB Dor. Figure 2 shows $\mathrm{H} \alpha$ observations of $\mathrm{AB}$ Dor for three successive nights. These show between three and five prominences in the observable part of the corona, some of which are clearly seen forming from night to night. The similarity in crossing times of the transients shows the prominences to be at similar distances from the rotation axis.

\section{Conclusions}

The ability to observe both the surface magnetic field and the positions of the prominences trapped high in the corona gives us a unique insight into the complex nature of the magnetic fields of rapidly-rotating stars. In the past, the ability to map surface features on single main sequence stars has been limited to nearby $\mathrm{K}$ stars. The use of least squares deconvolution presented here has allowed the sample of stars that can be studied in this way to be extended to G stars which have shallower convective regions. The use of these observations to produce surface maps of these $G$ stars will be the subject of further study.

Acknowledgments. The authors acknowledge the support of PPARC and the University of St. Andrews. One of the authors (MJ) completed this work while holding a PPARC Advanced Fellowship.

\section{References}

Collier Cameron, A. and Robinson, R.D. 1989a, MNRAS, 256, 57

Collier Cameron, A. and Robinson, R.D. 1989b, MNRAS, 238, 657

Collier Cameron, A. and Woods, J.A. 1992, MNRAS, 258, 360

Donati, J.-F. and Collier Cameron, A. 1997, MNRAS, 291, 1

Hendry, A., O'Dell, M.A. and Collier Cameron, A. 1993, MNRAS, 265, 983

O'Dell, M.A., Hendry, A. and Collier Cameron, A. 1994, MNRAS, 268, 181

Vogt, S.S. and Penrod, G.D., 1983, PASP, 95, 565 